\title{
Farnesoid $X$ receptor knockdown provides significant growth inhibition in hepatocellular carcinoma cells while it does not interfere with the proliferation of primary human hepatocyte-derived cells
}

\author{
Tomofumi Fujino', Akiko Maruko-Ohtake², Yosuke Ohtake², Tomonori Kobayashi², \\ Ken Ando', Airi Takeuchi', Yasuhito Ohkubo² and Makio Hayakawa1 \\ 'Department of Hygiene and Health Sciences, School of Pharmacy, Tokyo University of Pharmacy and Life Sciences, \\ 1432-1 Horinouchi, Hachioji 192-0392, Japan \\ ${ }^{2}$ Department of Radiopharmacy, Tohoku Pharmaceutical University, \\ 4-4-1 Komatsushima, Aoba-ku, Sendai, Miyagi 981-8558, Japan
}

(Received March 4, 2015; Accepted May 25, 2015)

\begin{abstract}
Identification of substances with specific toxicity for carcinoma cells promises to facilitate the development of cancer chemotherapeutics that cause minimal side effects. Here, we show that knockdown of the farnesoid X receptor (FXR) effectively suppresses the proliferation of human hepatocellular carcinoma cell lines HepG2 and HLE accompanied by elevated expression of cyclin-dependent kinase (CDK) inhibitor p16/INK4a and p21/Cip1 proteins. On the other hand, the growth of the primary human hepatocyte-derived cell line Fa2N-4 is not affected by the treatment with FXR siRNA irrespective of marked increases in the mRNAs of p16/INK4a and p21/Cip1. Surprisingly, the expression levels of p16/INK and p21/Cip1 proteins are left unchanged in Fa2N-4 cells that are subjected to the FXR siRNA treatment. Since the expression levels of these CDK inhibitor proteins in FXR-knockdown Fa2N-4 cells were elevated in the presence of proteasomal inhibitor MG132, these CDK inhibitors may be subjected to the proteasomal degradation, thereby counteracting the increased expression of their cognate mRNAs, therefore similar levels of p16 and p21 proteins were observed in control and FXR-knockdown Fa2N-4 cells. These results suggest that FXR-knockdown is effective for inhibiting the proliferation of hepatocellular carcinoma cells, not interfering with the regulatory mechanism of normal hepatocyte growth.
\end{abstract}

Key words: Farnesoid X receptor, Cyclin-dependent kinase inhibitors, Carcinoma-specific cytotoxicity

\section{INTRODUCTION}

Identification of substances that exhibit carcinoma cellspecific cytotoxicity will facilitate the development of cancer therapeutics that induces minimal side effects. We previously reported that the farnesoid X receptor (FXR), a bile acid-activated nuclear receptor (Makishima et al., 1999; Parks et al., 1999; Wang et al., 1999), stimulates the proliferation of hepatocellular carcinoma cell lines, HepG2 and HLE cells (Fujino et al., 2012). We found that RNA interference targeting FXR causes the elevation of cyclin-dependent kinase (CDK) inhibitor p16/INK4a expression in immunoblotting and quantitative PCR studies (Fujino et al., 2012). In contrast, activation of FXR results in the reduction of p16/INK4a expression in both mRNA and protein levels, thereby stimulating cell proliferation (Fujino et al., 2012). In this study, in order to examine whether or not FXR knockdown exerts carcinoma cell-specific cytotoxicity against hepatocellular carcinoma cells, we have evaluated the effect of FXR knockdown on the growth of simian virus 40 -immortalized cell line, Fa2N-4, which is derived from primary human hepatocytes. Additionally, we examined whether or not CDK inhibitor $\mathrm{p} 21 / \mathrm{Cip} 1$, also known as negative regulator of cell cycle (Sherr and Roberts, 1995), is involved into cytotoxicity of FXR knockdown on hepatocellular carcinoma cells.

Correspondence: Tomofumi Fujino (E-mail: tfujino@ps.toyaku.ac.jp) 


\section{T. Fujino et al.}

\section{MATERIALS AND METHODS}

\section{Materials}

Antibodies (Abs) specific for $\beta$-actin (C-2), FXR (D-3), p21 (H-164), and p16 (H-156) were purchased from Santa Cruz Biotechnology, Santa Cruz, CA, USA. MG132 was a product of Cell Signaling Technology, Danvers, MA, USA. GW4064 was from TOCRIS Bioscience, Ellisville, MO, USA. ECL ${ }^{\mathrm{TM}}$ anti-mouse $\mathrm{IgG}$, horseradish peroxidase linked whole antibody (from sheep) and $E L^{T M}$ anti-rabbit IgG, horseradish peroxidase linked whole antibody (from donkey) were purchased from GE Healthcare, Buckinghamshire, UK. Human hepatocellular carcinoma cell line, HepG2, HLE, and a primary human hepatocyte-derived cell line, Fa2N-4, were obtained from ATCC.

\section{Cell culture}

HepG2, HLE, and Fa2N-4 cells were maintained in Dulbecco's modified eagle medium (DMEM) containing $10 \%$ fetal calf serum (FCS), 50 units $/ \mathrm{mL}$ penicillin $\mathrm{G}$ sodium salt, and $50 \mu \mathrm{g} / \mathrm{mL}$ streptomycin sulfate and cultured in a humidified atmosphere of $8.5 \% \mathrm{CO}_{2}$ at $37^{\circ} \mathrm{C}$.

\section{RNA interference experiments}

The custom HP siRNA purchased from Qiagen K.K. (Tokyo, Japan) was used to knockdown the expression of FXR as described below.

According to the report by Sirvent et al. (2004), custom siRNA against FXR was prepared as follows: the mixture of the sense (5'-GUCGUGACUUGCGACAAGTT-3') and the anti-sense (5'-CUUGUCGCAAGUCACGACCT $-3^{\prime}$ ) oligonucleotides were denatured at $90^{\circ} \mathrm{C}$ and cooled down for annealing and used to knock down FXR. In order to knock down endogenous FXR, cells were seeded on 60 -mm dishes at a density of $4.5 \times 10^{5}$ cells (HepG2 and HLE) or $7.0 \times 10^{5}$ cells (Fa2N-4) per dish and transfected with siRNA against FXR (100 nM) using HiPerfect Transfection Reagent (Qiagen) according to the manufacturer's instructions. After incubating for 24,48 , or $72 \mathrm{hr}$, cell number was counted, and protein extracts for immunoblotting analyses or total RNAs for real-time PCR analyses were prepared. In the RNA interference experiments, "Nonsilencing Control" siRNA (\#1022076) from Qiagen was used as a control.

\section{Immunoblotting}

Cells were washed with PBS and cell extracts were prepared using SDS sample buffer without loading dye. After normalization of protein content by the protein assay, the dye was added to samples, followed by SDS-
PAGE and immunoblotting analysis. For the detection of FXR, p16, p21, and $\beta$-actin, the membranes were incubated with the primary $\mathrm{Ab}$ for $2 \mathrm{hr}$. Immunocomplexes on the PVDF membranes were visualized with enhanced chemiluminescence Western blotting detection reagents (GE Healthcare Biosciences).

\section{Quantification of mRNAs}

The amounts of mRNAs were quantified by realtime PCR. Briefly, $4 \mu \mathrm{g}$ of total RNAs were reversetranscribed by the use of ReverTra Ace qPCR RT Master Mix (Toyobo Co., Ltd. Life Science Department, Osaka, Japan) according to the manufacturer's instructions, and the resultant cDNAs were then subjected to real-time PCR analysis using TaqMan Gene Expression Assay kit (Life Technologies Japan, Tokyo, Japan). For the detection of FXR, p16, p21, and $\beta$-actin mRNAs, TaqMan assay mixtures Hs00231968, Hs99999189, Hs01121172, and 4310881E were used, respectively. TaqMan assay mixture Hs00231968 can detect all of the four isoforms of FXR (Zhang et al., 2004). Amplification and quantification were done with the PRISM 7000 RealTime PCR System (Life Technologies Japan). FXR, p16, and p21 mRNA levels were normalized to the levels of $\beta$-actin mRNA as an internal control. Data were analyzed by the Student's $t$-test.

\section{Measurement of DNA synthesis}

DNA synthesis was assessed by [methyl- $\left.{ }^{3} \mathrm{H}\right]$ thymidine incorporation into Fa2N-4 cells. [methyl- $\left.{ }^{3} \mathrm{H}\right]$ thymidine $(0.5 \mu \mathrm{Ci} / \mathrm{mL})$ incorporation in $\mathrm{Fa} 2 \mathrm{~N}-4$ was measured from 48 to $72 \mathrm{hr}$ after siRNA transfection or treatment with FXR ligand GW4064. After transfection or treatment with GW4064 for $72 \mathrm{hr}$, cells were washed in PBS, and solubilized in $2 \% \mathrm{NaOH}$. Radioactivity of [methyl${ }^{3} \mathrm{H}$ ] thymidine incorporated into cells was measured using a Beckman LS 6500 liquid scintillation counter (Beckman Coulter).

\section{RESULTS AND DISCUSSION}

\section{Knockdown of FXR elevates p21/Cip1 expression in hepatocellular carcinoma HepG2 and HLE cells}

In our previous study (Fujino et al., 2012), we proposed the mechanism that FXR acts as a regulator to stimulate the proliferation of HepG2 cells through the downregulation of p16/INK4a expression, by showing the data that FXR knockdown causes the elevation of p16/INK4a expression and suppresses cell growth whereas FXR activation with a synthetic ligand results in the 
Carcinoma-specific cytotoxicity by down-regulation of FXR

reduced expression of $\mathrm{p} 16 / \mathrm{INK} 4 \mathrm{a}$ accompanied by the increased cell number. In this study, we have revealed that FXR knockdown results in the elevation of not only p16/ INK4a expression but also p21/Cip1 expression in hepatocellular carcinoma cells. As shown in Fig. 1A, endogenous FXR expression in HepG2 or HLE cells became negligible when cells were subjected to the treatment with FXR siRNA. In those cells, expression levels of p21/Cip1 mRNA were significantly elevated (Fig. 1B). We further confirmed the remarkable increase in $\mathrm{p} 21 / \mathrm{Cip} 1$ protein detected by the anti-p21 Ab in FXR knockdown HepG2 cells (Fig. 1C). These results suggest that RNA interference targeting FXR can be the effective approach that potently suppresses the propagation of hepatocellular carcinoma by upregulating the expression of p16/INK4a and p21/Cip1, unless this approach interferes with the growth control of normal liver cells.

\section{Knockdown of FXR is not cytotoxic against primary human hepatocyte-derived Fa2N-4 cells}

To elucidate whether or not RNA interference targeting FXR provides the toxic effect on the growth of normal types of liver cells, we examined the growth of human hepatocyte-derived Fa2N-4 cells that are exposed to FXR siRNA. As shown in Fig. 2B and D, the growth and the $\left[{ }^{3} \mathrm{H}\right]$ thymidine incorporation of FXR-knockdown (Fig. 2A) cells was comparable to that of control cells. It should be noted that FXR knockdown does not influence the $\left[{ }^{3} \mathrm{H}\right]$ thymidine incorporation into primary cultured rat hepatocytes (data not shown). These results suggest that Fa2N-4 cells are phenotypically similar to rat primary hepatocytes in terms of proliferative controls that are insensitive to FXR knockdown, irrespective of being immortalized by Simian virus 40. Furthermore, GW4064, a synthetic ligand that potently activates FXR (Lew et al., 2004), did not stimulate the growth and the $\left[{ }^{3} \mathrm{H}\right]$ thymidine incorporation of Fa2N-4 cells (Fig. 2C and E), although it showed significant growth stimulation in HepG2 cells (Fujino et al., 2012). Thus we can postulate that FXR knockdown can selectively suppress the growth of hepatocellular carcinoma cells.

\section{FXR knockdown results in the upregulation of p16/INK4a and p21/Cip1 mRNAs; however, it does not lead to the increase in those CDK inhibitors at protein levels in Fa2N-4 cells}

In hepatocellular carcinoma cells including HepG2, RNA interference targeting FXR results in the increase in mRNA levels of CDKIs, thereby elevating the levels of their cognate proteins, p16/INK4a (Fujino et al., 2012) and p21/Cip1 (Fig. 1C). Surprisingly, FXR knock- down provided the significant increase in the mRNA levels of p16/INK4a and p21/Cip1 in Fa2N-4 cells (Fig. 3A) as observed in hepatocellular carcinoma cells. However, there was no difference in the expression levels of $\mathrm{p} 16 /$ INKa and p21/Cip1 proteins isolated from control and FXR-knockdown Fa2N-4 cells (Fig. 3B). Furthermore, irrespective of the significant decreases in the mRNA levels of p16/INK4a and p21/Cip in cells treated with the FXR ligand GW4064 (Fig. 3C), the expression levels of $\mathrm{p} 16 / \mathrm{INK} 4 \mathrm{a}$ or $\mathrm{p} 21 / \mathrm{Cip} 1$ proteins were comparable between control and GW4064-treated cells (Fig. 3D).

From these results, we can propose the role of FXR in the regulation of CDKIs as follows. FXR may play a role to downregulate the mRNA expression levels of $\mathrm{p} 16 /$ INK4a and p21/Cip1 in cells including normal hepatocytes and hepatocellular carcinoma cells. In those cells, lowered FXR expression by RNA interference results in the increased mRNA levels of these CDKIs. However, there seems to be a discrepancy between hepatocellular carcinoma cells and cells derived from primary hepatocytes in the regulation of CDKIs expression at posttranscriptional level, $i$. e., the increase in each CDKI mRNAs caused by FXR knockdown directly leads to the elevated expression of their cognate proteins in the former while it fails to increase them in the latter. From these results, we postulate that primary hepatocytes retain the regulatory system that leads to the decrease in stability of CDKI proteins whereas certain types of hepatocellular carcinoma cells have lost this system thereby FXR knockdown effectively upregulates CDKIs.

\section{Proteasomal degradation accounts for the posttranscriptional regulation of CDKIs that are not upregulated in primary hepatocyte-derived Fa2N-4 cells exposed to FXR knockdown}

The ubiquitin-proteasome system plays a pivotal role in the sequence of events by leading to the degradation of key molecules such as cyclins and p57/Kip2 during the cell division cycle (Teixeira and Reed, 2013). Given that stability of CDKI proteins was decreased at FXR-knockdown Fa2N-4 cells, we next examined whether or not the proteasome is involved in the degradation of $\mathrm{p} 16 / \mathrm{INK} 4 \mathrm{a}$ and p21/Cip1 proteins in FXR siRNA-treated Fa2N-4 cells. As shown in Fig. 4A, immunoblotting analyses revealed that the levels of p16/INK4a and p21/Cip1 in control Fa2N-4 cells treated with the proteasomal inhibitor MG132 (Lee and Goldberg, 1998) were comparable to that in control cells left untreated with MG132. In contrast, MG132 treatment caused the significant increases in both p16/INK4a and p21/Cip1 proteins in FXR knockdown Fa2N-4 cells, in which mRNAs of these CDKIs 
T. Fujino et al.

A

\section{HepG2 cells}

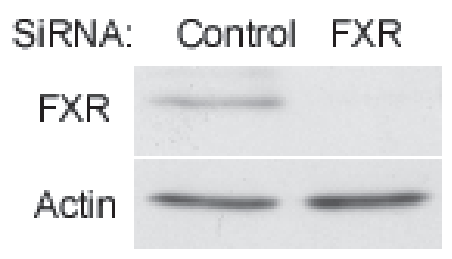

B

HepG2 cells

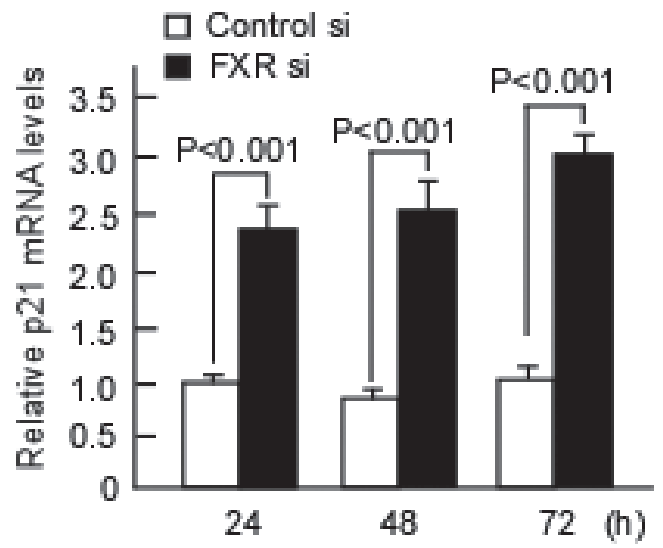

\section{HLE cells}

SiRNA: Control FXR

FXR

Actin

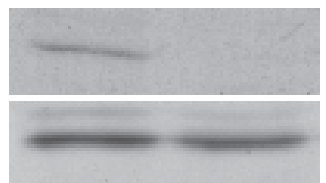

\section{HLE cells}

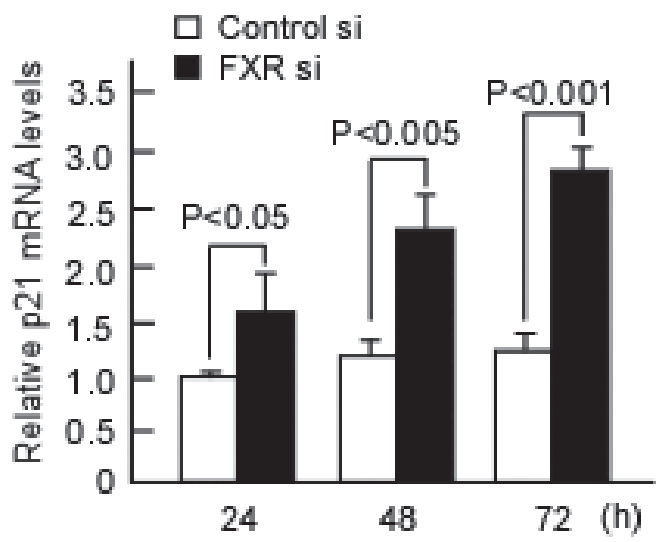

C HepG2 cells

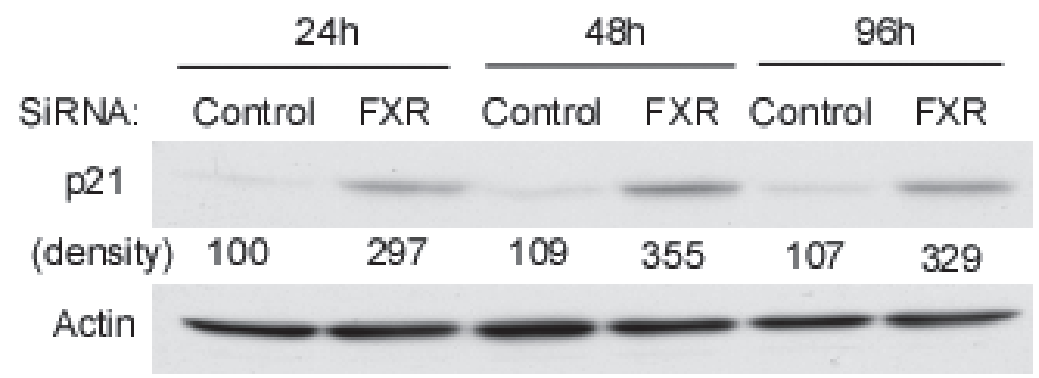

Fig. 1. Knockdown of FXR results in the increase in p21/Cip1 expression in hepatocellular carcinoma HepG2 and HLE cells. Hepatocellular carcinoma cells seeded at a density of $4.5 \times 10^{5}$ cells per $60-\mathrm{mm}$ dish were transfected with a control siRNA or with an siRNA against human FXR and cultured for $72 \mathrm{hr}$. (A) Total protein extracted from HepG2 and HLE cells was subjected to immunoblotting analyses to detect FXR and $\beta$-actin. (B) Total RNA was extracted from HepG2 and HLE cells at the indicated times and subjected to real-time RT-PCR analysis to quantify p21/Cip1 mRNA levels. Data are shown as the mean \pm S.D. of four determinations and analyzed via a Student's t-test. (C) Total protein was extracted from HepG2 cells at the indicated times and subjected to immunoblotting analyses to detect p21/Cip1 and $\beta$-actin. 
Carcinoma-specific cytotoxicity by down-regulation of FXR

\section{A}

SiRNA: Control FXR

FXR

Actin

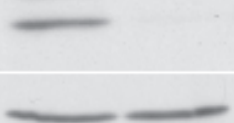

B

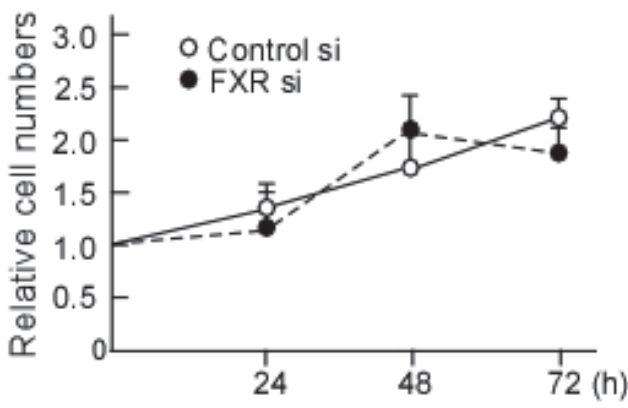

C

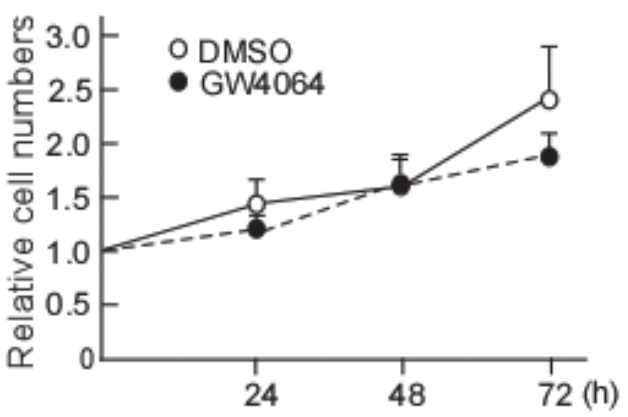

D

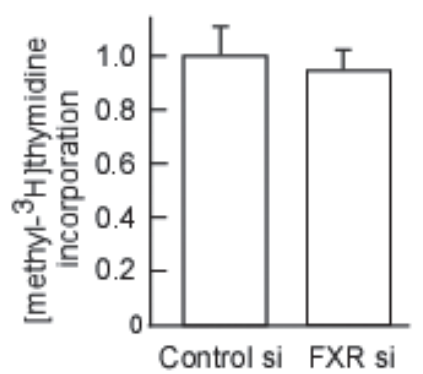

$E$

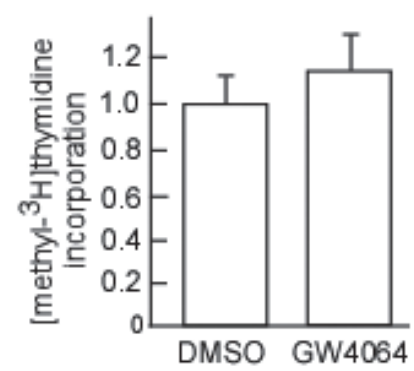

Fig. 2. Neither suppression nor activation of FXR affects the proliferation of Fa2N-4 cells derived from human primary hepatocytes. (A) Fa2N-4 cells seeded at a density of $7.0 \times 10^{5}$ cells per $60-\mathrm{mm}$ dish were transfected with a control siRNA or with an siRNA against human FXR and cultured for $72 \mathrm{hr}$. Then cells were subjected to the immunoblotting analysis as described in Fig. 1A. (B and D) Fa2N-4 cells seeded at a density of $7.0 \times 10^{5}$ cells per $60-\mathrm{mm}$ dish were transfected with a control siRNA or with an siRNA against human FXR for $72 \mathrm{hr}$. Cell number was determined at indicated times (B). [methyl- ${ }^{3} \mathrm{H}$ ] thymidine incorporation in cells was measured from 48 to $72 \mathrm{hr}$ after siRNA transfection (D). Data are shown as the mean \pm S.D. of four determinations. (C and E) Fa2N-4 cells seeded at a density of $7.0 \times 10^{5}$ cells per $60-\mathrm{mm}$ dish were treated with DMSO as a control or with $5 \mu \mathrm{M} \mathrm{GW} 4064$ for $72 \mathrm{hr}$. Cell number was determined at indicated times $(\mathrm{C})$. [methyl $\left.{ }^{3} \mathrm{H}\right]$ thymidine incorporation in cells was measured from 48 to $72 \mathrm{hr}$ after siRNA transfection (E). Data are shown as the mean \pm S.D. of four determinations. 
A

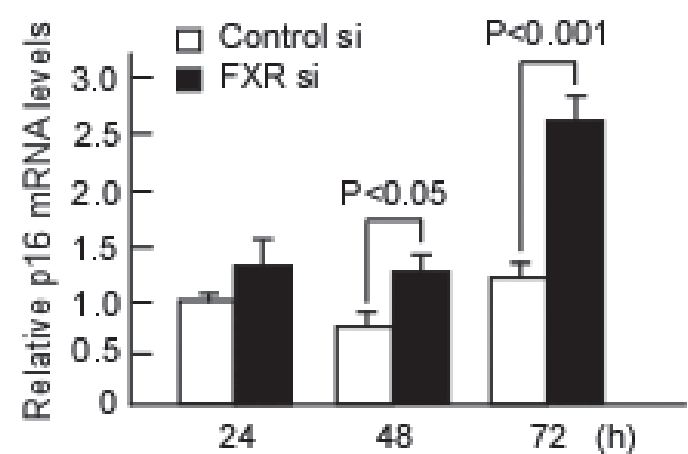

B

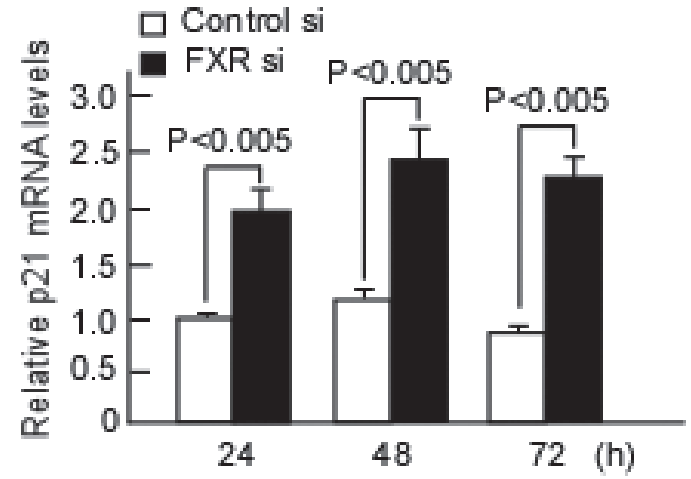

24

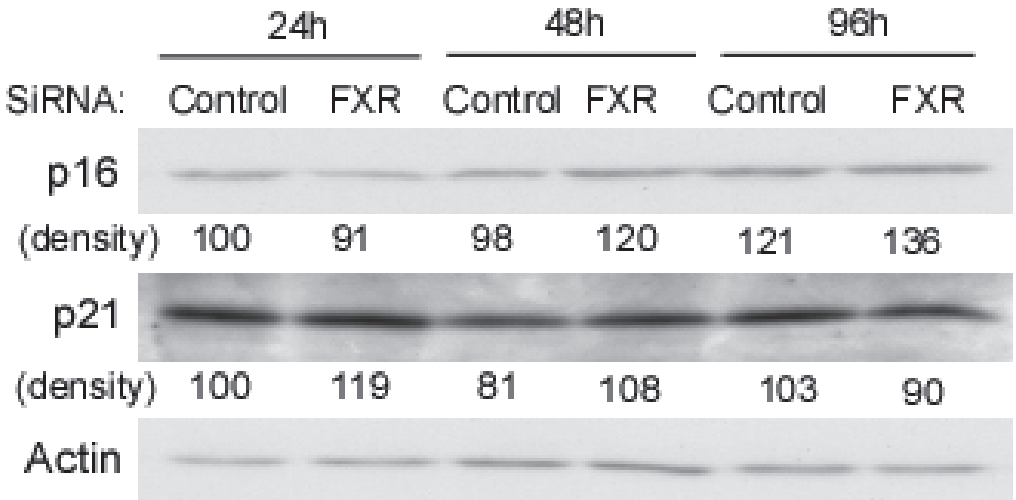

C

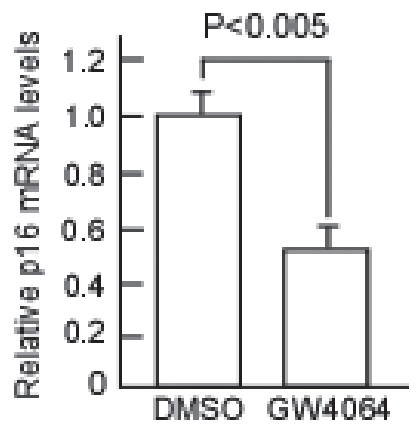

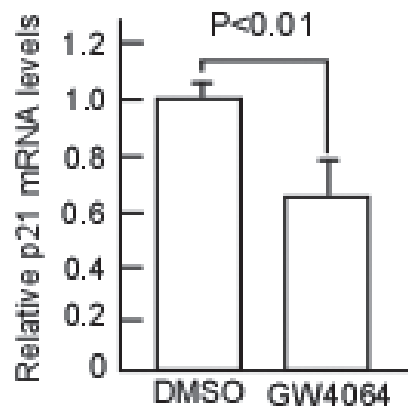

D

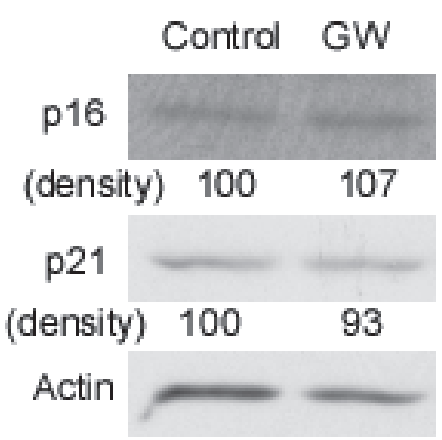

Fig. 3. Regulation of CDKIs expression at posttranscriptional level is uncoupled from that of their cognate mRNA levels in Fa2N-4 cells in which FXR is suppressed or activated. (A) Fa2N-4 cells seeded at a density of $7.0 \times 10^{5}$ cells per 60 -mm dish were transfected with a control siRNA or with an siRNA against human FXR and cultured for $72 \mathrm{hr}$. Total RNA was extracted at the indicated times and subjected to real-time RT-PCR analysis to quantify p16/INK4a or p21/Cip1 mRNA levels. Data are shown as the mean \pm S.D. of four determinations and analyzed via a Student's t-test. (B) As described in A, Fa2N-4 cells were seeded and transfected with the indicated siRNAs, then subjected to the immunoblotting analyses to detect p16/ INK4a, p21/Cip1, and $\beta$-actin. (C) Fa2N-4 cells seeded at a density of $7.0 \times 10^{5}$ cells per 60 -mm dish were treated with DMSO as a control or with $5 \mu \mathrm{M}$ GW4064 and cultured for $72 \mathrm{hr}$, then total RNA was extracted and subjected to real-time RT-PCR analysis to quantify p16/INK4a or p21/Cip1 mRNA levels. Data are shown as the mean \pm S.D. of four determinations and analyzed via a Student's t-test. (D) As described in C, Fa2N-4 cells were treated with GW4064 then subjected to the immunoblotting analyses to detect p16/INK4a, p21/Cip1, and $\beta$-actin. 
Carcinoma-specific cytotoxicity by down-regulation of FXR

A

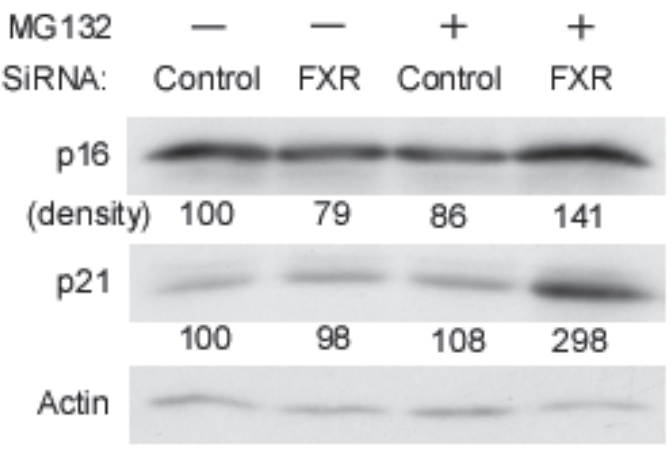

B

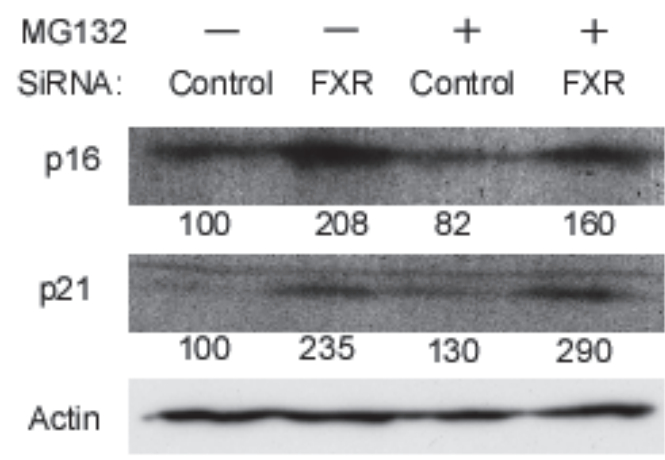

Fig. 4. Possible involvement of the proteasome in the downregulation of CDKIs in Fa2N4 cells in which FXR expression is suppressed. (A) Fa2N-4 cells seeded at a density of $7.0 \times 10^{5}$ cells per 60 -mm dish were transfected with a control siRNA or with an siRNA against human FXR and cultured for $72 \mathrm{hr}$ in the presence or absence of $10 \mu \mathrm{M}$ MG132. Immunoblotting analyses were performed to detect p16/INK4a, p21/Cip1, and $\beta$-actin, respectively. Quantification of the bands was done by densitometric analysis (IMAGE GAUGE 4.0). (B) HepG2 cells seeded at $4.5 \times 10^{5}$ cells per $60-\mathrm{mm}$ were subjected to the immunoblotting analyses as described in A.

were elevated. From these results we can postulate that p16/INK4a and p21/Cip1 do not undergo the proteasomal degradation when cells express FXR whereas they become recognized by the proteasome in cells in which FXR expression is significantly suppressed. Interestingly, in neither control nor FXR-knockdown HepG2 cells, MG132-dependent increases in p16/INK4a and p21/Cip1 were observed (Fig. 4B). In the case of this hepatocellular carcinoma cells, degradation pathway mediated through the proteasome may show the marginal effect on the regulation of p16/INK4a and p21/Cip1, therefore, FXR- knockdown-dependent elevation of these CDKI mRNAs is reflected as the increased expression of their cognate proteins in the presence or absence of MG132.

There are several reports describing the degradation of p16/INK4a or p21/Cip1 mediated through the ubiquitinproteasome system (Roberti et al., 2011; Han et al., 2013). Although we could not detect the p16/INK4a or p21/Cip1 bands accompanying high molecular weight ladder in the sample derived from FXR knockdown Fa2N-4 cells treated with MG132 under the present experimental condition, it is still possible that polyubiquitination of these CDKIs proceeds in response to the step-down expression of FXR in these cells.

On the other hand, ubiquitination-independent degradation mediated through the REG $\gamma$ proteasome has been shown to contribute to the downregulation of $\mathrm{p} 21 / \mathrm{Cip}$ and p16/INK4a (Chen et al., 2007; Li et al., 2007). Although the precise mechanism how p21/Cip and p16/INK4a are recognized by REG $\gamma$ (also known as PA28) that binds and activates $20 \mathrm{~S}$ proteasome remains to be elucidated, it is worthwhile to examine whether or not the REG $\gamma$ proteasome is responsible for the degradation of p21/Cip and p16/INK4a in Fa2N-4 cells in which FXR expression is interfered.

By focusing on the FXR-dependent regulation of CDKIs expression, we have succeeded in showing that the interference of FXR expression can be useful for the selective suppression of hepatocellular carcinoma propagation. Further insights will be directed into the following molecular basis in the future studies: 1) how FXR regulates the expression of CDKI mRNAs, 2) which type of the proteasomal pathway is responsible for the downregulation of CDKIs in normal liver cells, 3) why the proteasomal pathway responsible for CDKIs downregulation is impaired in hepatocellular carcinoma cells.

\section{ACKNOWLEDGMENTS}

We thank Toshiaki Tanaka for helpful advice and discussions. This work was supported in part by a grant from the Japan Private School Promotion Foundation.

Conflict of interest---- The authors declare that there is no conflict of interest.

\section{REFERENCES}

Chen, X., Barton, L.F., Chi, Y., Clurman, B.E. and Roberts, J.M. (2007): Ubiquitin-independent degradation of cell-cycle inhibitors by the REGgamma proteasome. Mol. Cell, 26, 843-852.

Fujino, T., Takeuchi, A., Maruko-Ohtake, A., Ohtake, Y., Satoh, J., Kobayashi, T., Tanaka, T., Ito, H., Sakamaki, R., Kashimura, R., 
T. Fujino et al.

Ando, K., Nishimaki-Mogami, T., Ohkubo, Y., Kitamura, N., Sato, R., Kikugawa, K. and Hayakawa, M. (2012): Critical role of farnesoid $\mathrm{X}$ receptor for hepatocellular carcinoma cell proliferation. J. Biochem., 152, 577-586.

Han, J., Kim, Y.-L., Lee, K.-W., Her, N.-G., Ha, T.-K., Yoon, S., Jeong, S.-I., Lee, J.-H., Kang, M.-J., Lee, M.-G., Ryu, B.-K., Baik, J.-H. and Chi, S.-G. (2013): ZNF313 is a novel cell cycle activator with an E3 ligase activity inhibiting cellular senescence by destabilizing p21 ${ }^{\text {WAF1 }}$. Cell Death Differ., 20, 1055-1067.

Lee, D.H. and Goldberg, A.L. (1998): Proteasome inhibitors: valuable new tools for cell biologists. Trends Cell Biol., 8, 397-403.

Lew, J.L., Zhao, A., Yu, J., Huang, L., De Pedro, N., Peláez, F., Wright, S.D. and Cui, J. (2004): The farnesoid X receptor controls gene expression in a ligand- and promoter-selective fashion. J. Biol. Chem., 279, 8856-8861.

Li, X., Amazit, L., Long, W., Lonard, D.M., Monaco, J.J. and O'Malley, B.W. (2007): Ubiquitin- and ATP-independent proteolytic turnover of p21 by the REGgamma-proteasome pathway. Mol. Cell, 26, 831-842.

Makishima, M., Okamoto, A.Y., Repa, J.J., Tu, H., Learned, R.M., Luk, A., Hull, M.V., Lustig, K.D., Mangelsdorf, D.J. and Shan, B. (1999): Identification of a nuclear receptor for bile acids. Science, 284, 1362-1365.

Parks, D.J., Blanchard, S.G., Bledsoe, R.K., Chandra, G., Consler, T.G., Kliewer, S.A., Stimmel, J.B., Willson, T.M.,
Zavacki, A.M., Moore, D.D. and Lehmann, J.M. (1999): Bile acids: natural ligands for an orphan nuclear receptor. Science, 284, 1365-1368.

Roberti, A., Rizzolio, F., Lucchetti, C., de Leval, L. and Giordano, A. (2011): Ubiquitin-mediated protein degradation and methylation-induced gene silencing cooperate in the inactivation of the INK4/ARF locus in Burkitt lymphoma cell lines. Cell Cycle, 10, 127-134.

Sherr, C.J. and Roberts, J.M. (1995): Inhibitors of mammalian G1 cyclin-dependent kinases. Genes Dev., 9, 1149-1163.

Sirvent, A., Verhoeven, A.J., Jansen, H., Kosykh, V., Darteil, R.J., Hum, D.W., Fruchart, J.C. and Staels, B. (2004): Farnesoid X receptor represses hepatic lipase gene expression. J. Lipid Res., 45, 2110-2115.

Teixeira, L.K. and Reed, S.I. (2013): Ubiquitin Ligases and Cell Cycle Control. Annu. Rev. Biochem., 82, 387-414.

Wang, H., Chen, J., Hollister, K., Sowers, L.C. and Forman, B.M. (1999): Endogenous bile acids are ligands for the nuclear receptor FXR/BAR. Mol. Cell, 3, 543-553.

Zhang, Y., Castellani, L.W., Sinal, C.J., Gonzalez, F.J. and Edwards, P.A. (2004): Peroxisome proliferator-activated receptor-gamma coactivator 1alpha (PGC-1alpha) regulates triglyceride metabolism by activation of the nuclear receptor FXR. Genes Dev., 18, 157-169. 